\title{
Anabases
}

ANABASES Traditions et réceptions de l'Antiquité

$3 \mid 2006$

Varia

\section{Enseigner autrement les Humanités classiques en Europe : propositions à partir du cas français}

\section{Stéphane Ratti}

\section{(2) OpenEdition}

\section{Journals}

Édition électronique

URL : http://journals.openedition.org/anabases/2733

DOI : 10.4000 /anabases. 2733

ISSN : 2256-9421

Éditeur

E.R.A.S.M.E.

\section{Édition imprimée}

Date de publication : 1 mars 2006

Pagination : 215-233

ISSN : 1774-4296

\section{Référence électronique}

Stéphane Ratti, «Enseigner autrement les Humanités classiques en Europe : propositions à partir du cas français », Anabases [En ligne], 3 | 2006, mis en ligne le 01 janvier 2012, consulté le 21 octobre 2019. URL : http://journals.openedition.org/anabases/2733 ; DOI : 10.4000/anabases.2733 
Anabases 3 (2006), p. 215-233

\section{Enseigner autrement les Humanités classiques en Europe : propositions à partir du cas français}

STÉPHANE RATTI

\section{Préambule}

L' INTÉRÊT POUR L'ANTIQUiTÉ gréco-romaine n'a jamais été aussi fort en France. L'attestent les nombreux voyages scolaires organisés par les établissements secondaires (Grèce, Rome, Pompéi, Sicile, Afrique du Nord), les échanges entre établissements et familles, le développement des clubs de lecture et d'apprentissage du grec ancien. La vitalité des publications en traduction des auteurs antiques constitue un autre signe de cet intérêt pour l'Antiquité. C'est donc peu de dire que ce mouvement se développe à une époque où la recherche des racines et la réflexion sur les thèmes liés à la citoyenneté connaissent un nouvel élan. La place de la culture générale dans les formations universitaires est aujourd'hui l'objet d'une réflexion approfondie menée au sein de la Mission d'Étude et de Proposition créée en juillet $2001{ }^{1}$. Une autre mission ministérielle a cherché parallèlement à redéfinir les objectifs des Humanités classiques en liaison notamment avec la perspective de la constitution d'un socle culturel commun aux pays de l'Europe élargie ${ }^{2}$.

Il y a aujourd'hui en France (année scolaire 2003-2004 3) 60000 latinistes dans les lycées et 32000 hellénistes au total. Dans les collèges, le latin est offert en option en classe de Cinquième depuis la réforme Bayrou de 1995. Le succès de cette option est incontestable, puisque un élève sur cinq la choisit. On compte au total 460000 élèves latinistes sur les trois niveaux du collège. Les besoins en professeurs de lettres classiques sont réels pour les années à venir.

Néanmoins cette évolution historique est marquée par un paradoxe : l'intérêt intellectuel pour l'Antiquité classique va de pair avec une désaffection, dans les lycées, pour des options de langues anciennes jugées peu rentables ou difficiles d'accès en raison de multiples freins administratifs. Il paraît dès lors nécessaire de rappeler quelques principes :

1 Sous la direction d'A. Renaut. Le colloque " Mettre les savoirs en culture " s'est tenu en Sorbonne le 20 mai 2003.

2 Sous la direction de H. WismanN.

3 Source : P. SOLER, "Rapport de l'Inspection générale", Actes du XXXVI Congrès de l'Association des professeurs de langues anciennes de l'enseignement supérieur, Pau, 2004, p. 162-163. 
- les langues anciennes n'ont pas d'utilité langagière immédiate ; cet enseignement place donc parmi ses priorités l'acquisition synthétique des catégories linguistiques et lexicales, en particulier les vocabulaires spécialisés et techniques. Cette étude distanciée (latin et grec sont à la fois proches et lointains) facilite une nouvelle approche de la langue maternelle mais aussi celui des autres langues vivantes, romanes ou non. Les langues anciennes sont au service d'un objectif fondamental : communiquer clairement. On sait aussi que la dissertation traditionnelle doit son plan aux catégories élaborées par Cicéron. En ce sens, la connexion de l'enseignement des Lettres classiques avec la littérature française est forte et doit être maintenue ;

- le système éducatif doit se donner les outils nécessaires pour former chez les élèves une capacité critique permettant à la fois une sélection des informations véhiculées - à un débit de plus en plus rapide - par les textes et les images, l'analyse et la synthèse de leur contenu. Les langues anciennes permettent l'acquisition systématique de ces modes de pensée fondamentaux qui s'exercent, dans nos études, sur des textes et des images de haute valeur culturelle ;

- la maitrise de ces outils de base et de ces catégories réutilisables dans d'autres domaines autorisera les reconversions et les adaptations ; les acquis faciliteront les accommodations et les évolutions au fil des contraintes économiques et professionnelles sans cesse en mouvement. Les Universités ont ainsi parfois mis en place des formations innovantes destinées à préparer les étudiants à d'autres débouchés que ceux de l'enseignement. Elles rencontrent ainsi les souhaits de nombreux chefs d'entreprise heureux de mettre en œuvre les capacités de synthèse et d'analyse des littéraires ainsi que leurs compétences dans le domaine de l'expression et de l'organisation.

Ces objectifs supposent la poursuite et l'intensification des efforts d'adaptation et de modernisation pédagogiques entrepris depuis plusieurs années dans l'enseignement secondaire et supérieur. Ces efforts peuvent-ils prendre appui sur les textes officiels qui encadrent l'enseignement des langues anciennes? Quelles perspectives élargies ces textes ouvrent-ils ? Peuvent-ils contribuer à une revalorisation nécessaire des Humanités classiques ? C'est à ces questions que je voudrais répondre en proposant une lecture critique articulée en trois étapes : la notion de genre littéraire est-elle pertinente pour aborder la littérature latine ? quels sont les contours précis de l'héritage antique? qu'est-ce que lire un texte ancien?

\section{La notion de genre littéraire est-elle pertinente pour aborder la littérature latine?}

Le genre est un concept qui structure les Instructions Officielles $(I O)$ de lycée : le mot apparaît à plusieurs reprises dans les $I O$ dès la classe de Seconde ${ }^{4}$ et réapparaît en Première et Terminale ${ }^{5}$. Le programme de seconde est ainsi libellé : "Textes, genres et références historiques et culturelles " (p. 3). La lecture des sous-titres est instructive :

4
Bulletin Officiel du 29 août 2002.

Cf. p. 7 du Journal Officiel du 4 août 2001 pour la classe de Terminale : «L'étude des genres » est placée au centre des " apprentissages et de la progression » et le Bulletin Officiel hors série $\mathrm{n}^{\circ} 3 \mathrm{du} 30$ août 2001 (programme de la classe de Terminale) ; cf., pour les nouvelles épreuves de latin et de grec du baccalauréat, le Bulletin Officiel $\mathrm{n}^{\circ} 21$ du 22 mai 2003. Cf. aussi l'excellent Langues anciennes. Guide pédagogique pour le professeur, 2005, Eduscol, en ligne, de P. Charvet et P. Soler. 
1. Éloquence judiciaire et politique : un grand orateur Cicéron

2. Comédie, satire, épigramme

3. Histoire et épopée.

\section{L'éloquence judiciaire et politique : un genre unique?}

À y regarder de près, que signifient ces sous-titres ? Le premier point mêle «éloquence judiciaire » et " éloquence politique " autour de Cicéron, comme si la forme l'emportait sur le contenu, l'expression sur l'objectif, la catégorie linguistique sur le projet humain : l'éloquence est objet d'étude plutôt que le procès de Milon, la vie du barreau l'emportant sur la condamnation ou la relaxe de tel accusé ; la rhétorique est objet d'étude plutôt que la crise politique née du meurtre de Clodius. En réalité, le risque existe ici de négliger l'inscription toujours très forte de la rhétorique latine dans un contexte - inscription toujours prégnante, sauf peut-être dans le cas extrême des suasoires d'école telles que Sénèque le Père nous les a transmises, même dans les panégyriques des III et IVe siècles où une lecture rapide ne sait pas toujours la dépister sous les oripeaux de la flagornerie. L'éloquence est toujours inscrite dans une réalité politique et sociale, et l'oublier serait glisser dans un formalisme (la Rhétorique comme essence) inapplicable à la chose romaine. En bref, la question qui se pose à la lecture du sous-titre évoqué est la suivante : existe-t-il une rhétorique qui subsume l'éloquence judiciaire et l'éloquence politique ou encore : ces deux éloquences sontelles une seule et même chose?

En réalité, il existe une différence considérable entre le Cicéron des Verrines (Verrès fait l'objet d'une accusation de droit commun) et celui des Catilinaires (Catilina est poursuivi par la verve de Cicéron pour des motifs politiques). Attardons-nous un instant sur les Verrines. Dans ces discours, prononcés en 70, un homme jeune (Cicéron a 36 ans) et un ancien questeur inconnu des milieux politiques (il est au début de son cursus dont il a tout juste franchi les premiers échelons, précisément en Sicile, à Lilybée) accuse de concussion un homme puissant, le propréteur d'une importante province, la Sicile. Ce qui frappe ici c'est la démesure de l'entreprise : un homme dont la famille n'a jamais exercé le consulat ose s'attaquer à un notable ; un modeste avocat sans réputation pense faire citer plus de mille témoins à charge ; un jeune ambitieux sans curriculum uitae passe au crible la carrière d'un homme en place : dénonciation publique des tricheries de Verrès dans sa fonction juridique (De praetura Siciliensi), dans sa perception des impôts (De re frumentaria), dans ses vols d'œuvres d'art (De signis), dans ses abus de pouvoir (De suppliciis). La rhétorique des Verrines est marquée par cette démesure et ne se cantonne pas à un seul registre mais use du mélange des genres. Se succèdent dans ces discours des narrations et des descriptions tandis que le ton change en permanence : on oscille ainsi entre pathétique et humour (souvent facile), entre ironie et virulence (parfois triviale). On est loin de l'uniformité de ton d'un discours d'Isocrate et ce serait une gageure que de tenter une codification de la rhétorique latine à partir des seules Verrines, qui sont autant un pamphlet contre les excès auxquels mène l'exercice déréglé du pouvoir solitaire qu'une description psychologique orientée de la passion d'un maniaque. Les Verrines de Cicéron ne permettent sans doute pas davantage que le Pro Milone de circonscrire les limites génériques exactes de la rhétorique judiciaire à l'époque de Cicéron en raison de leur polymorphisme.

Si un discours évidemment catalogué par la tradition - et cela ne doit pas être contesté dans le genre de l'éloquence judiciaire n’offre pas de réelle unité de ton, peut-on rechercher cette 
unité dans l'éloquence cicéronienne sans tenir compte des sous-genres " juridique » et " politique " ? En d'autres termes, y a-t-il un point commun fort entre les Verrines et les Catilinaires qui justifie leur inclusion dans une même rubrique? Je ne le crois pas. En effet, si le Cicéron de 70 est un inconnu, celui de 63 est consul. L'homo nouus a fait du chemin depuis Lilybée. Le poids de la parole cicéronienne (sa grauitas, au sens étymologique) tombe à cette date de beaucoup plus haut et c'est au nom de l'État que s'exprime aujourd'hui celui dont on ne savait pas, quelques années plus tôt, "d'où il parlait ». Catilina est présenté comme l'ennemi public afin de façonner l'image d'un sauveur public, celui qui portera dès lors le titre si rare de pater patriae, souvenir des mots par lesquels, aux dires de Tite-Live, on saluait Romulus, parens patriae. Ce que veut faire Cicéron, c'est hausser sa propre gloire, mais aussi, à travers un épisode présenté comme une crise nationale, démontrer que le pouvoir doit naturellement revenir aux intellectuels éclairés, dont il estime faire partie. C'est toute la crise de la Révolution romaine, pour reprendre le titre de Ronald Syme (1939), que l'on peut ici appréhender à travers les Catilinaires. Cicéron a pressenti la montée en puissance des imperatores et a cherché, à travers sa propre personne, à montrer qu'un simple civil cultivé et bon rhéteur - c'est-à-dire en fait un citoyen idéalement préparé par la pratique de la rhétorique - était apte à exercer le pouvoir pour le bien de la Res publica. Le vers fameux par lequel Cicéron décrivait son programme politique dans le poème qu'il avait écrit sur son propre consulat n'a pas d'autre signification : Cedant arma togae, concedat laurea laudi, "Que les armes s'effacent devant la toge, que le laurier s'incline devant la gloire ». Cicéron a poussé un cri d'alarme et pressenti les catastrophes qui allaient accompagner les conflits civils encore à venir.

On le voit, la rhétorique des Catilinaires, si elle emprunte au même arsenal formel, n'a, ni dans ses ambitions ni dans ses conséquences, rien de commun avec celle des Verrines. Le projet des secondes n'est pas moins ambitieux que celui des premières, mais l'homme qui les a toutes deux formulées n'occupe pas dans l'espace politique romain la même surface. Les deux séries de discours peuvent être lues comme l'analyse des vices qui un jour saperont les fondements de la libera res publica, mais le point commun à ces deux œuvres ne saurait être ramené à leur appartenance au genre de l'éloquence. La distinction entre le genre judiciaire (on plaide devant un tribunal) et le genre délibératif (on parle devant une assemblée) s'efface à Rome plus souvent qu'on ne le pense. L'absence de huis clos et le parallèle avec nombre de procès retentissants à notre époque doivent faire comprendre la facilité avec laquelle une affaire judiciaire peut devenir politique.

On doit donc douter de la pertinence des termes "éloquence judiciaire et politique " pour désigner un genre unique. Une saine approche de la rhétorique antique ne saurait être exclusivement formaliste, sauf à restreindre l'éloquence à un ensemble de recettes ou de techniques. La rhétorique n'est surtout pas assimilable à un catalogue de tropes ni à un dictionnaire de figures. C'est un genre duquel dérivent tous les autres genres, par exemple l'historiographie ${ }^{6}$ et la philosophie ${ }^{7}$, sans parler de l'épopée (et donc du roman). Sans exagération, on peut même affirmer

6 Sur ce sujet, on lira par exemple J. DANGEL, "Les Muses de l'histoire : l'énigme cicéronienne", dans Grecs et Romains aux prises avec l'histoire. Représentations, récits et idéologie, éd. G. Lachenaud et D. Longrée, Presses Universitaires de Rennes, 2003, vol. I, p. 8595 , ici p. 88.

7 C'est vrai depuis Isocrate au moins, pour qui le logos est la connaissance. 
que toute la littérature moderne est issue de la rhétorique, d'abord celle qui a cherché à convaincre (héritage d'une pratique politico-judiciaire), puis, sans doute à partir du Romantisme, celle qui a cherché par les mots à atteindre un ailleurs de la Raison ${ }^{8}$. La rhétorique est autant une pratique sociale et un mode de réflexion intellectuel qu'un genre au sens strict.

Il s'agit aussi d'un enseignement. Sans doute est-ce ce que les $I O$ veulent signifier par ce regroupement autour de Cicéron, celui qui a codifié pour longtemps (avec l'aide plus tard du très pragmatique Quintilien), notamment dans le De oratore, les fondements de l'enseignement moderne. Les exordes des première et deuxième Catilinaires figurent au programme des lycées. $\mathrm{Ne}$ pourrait-on envisager une lecture (plus) transversale, par exemple avec une problématique telle que "Forme et fonction de l'exorde " ? On verrait alors que l'exorde vaut expositio (annonce du plan), captatio beneuolentiae (séduction du lecteur) et refutatio (réponse anticipée aux arguments adverses). Mettez " correcteur " à la place de "lecteur " et vous aurez les règles toujours actuelles de la dissertation moderne. Cicéron est en effet le Père (avec un fonds philosophique emprunté à Aristote) des catégories formelles de l'expression écrite telle que la pratique idéalement l'institution scolaire.

Une dernière observation me vient à l'esprit à propos de l'intitulé "Un grand orateur Cicéron ». On évoque ici un monument monolithique, un Cicéron d'une pièce, comme statufié. En réalité, la personnalité même de Cicéron fait question, ce qui ne doit pas être dissimulé. Tout d'abord, il faut avoir conscience que loin d'avoir été un simple rhéteur, Cicéron a illustré tous les genres et avec brio (philosophie ; littérature épistolaire ; poésie : il n'y a guère que l'historiographie à laquelle Cicéron, qui en avait néanmoins le projet, ne se soit pas consacré comme praticien). Son nom devrait ainsi plutôt servir à illustrer la volonté totalitaire de son écriture. Il n'y a pas qu'un Cicéron et surtout pas le Cicéron dont les $I O$ donnent une image d'Épinal : «Un homme politique courageux et un magnifique orateur ${ }^{9}$..."

L'image est flatteuse (comme si l'approche scolaire devait absolument passer par la phase de l'admiration), mais surtout douteuse. La vérité historique, et sans doute aussi la vertu attractive du personnage, auraient tout à gagner si l'on rappelait que Cicéron n'a pas toujours été courageux. On connaît ses difficultés à adopter un parti, ses palinodies et sa peur de César. Tite-Live dit de lui : omnium aduersorum nibil ut uiro dignum erat tulit praeter mortem (" parmi tous les malheurs qui lui sont arrivés il n'en supporta aucun comme aurait dû le faire un homme, sauf sa mort $10 »)$. Cicéron n'a pas non plus toujours été un " orateur magnifique » : on sait combien piètre fut sa prestation bafouillée en 52 lors du procès de Milon auquel il n'a pas su éviter la relégation marseillaise. Tout aussi pertinent serait le parti de révéler le Cicéron hésitant, torturé, dupé, lâche ou encore le républicain tantôt enthousiaste, nourri d'illusions sur la restauration en cours de la Res publica (en 46, dans le Pro Marcello), tantôt désespéré (plus tôt, à la mort de Pompée). On peut tout aussi bien souhaiter privilégier le Cicéron humain tel que le révèlent les 900 lettres d'une correspondance exceptionnellement riche. Sans aller aussi loin que Jérôme

8 C'est ce que Roland BARTHES appelle "L'empire rhétorique » dans son étude fondamentale, "L'ancienne rhétorique", publié initialement dans Communications 16, 1970, et repris dans Euvres complètes, vol. III, Paris, 2002, p. 527-601, ici p. 530.

10 Tite-Live, fragment 59 (livre 120) = Sénèque le Père, Suasoriae 6, 17. 
Carcopino ${ }^{11}$ qui faisait de Cicéron, chapitre après chapitre, " un mari trop intéressé ", " un père trop indifférent ", " un beau-père trop accommodant " et j'en passe, l'analyse littéraire pourrait se focaliser autour de l'objectif suivant : "La mise en scène de soi dans l'œuvre de Cicéron ». Cet homme sait tout faire : il donne des conseils sur la manière de se soigner à son affranchi Tiron, tout en dépréciant les prescriptions d'un médecin spécialiste (sed plane curationes eius non probo, «Mais je n'approuve absolument pas le traitement qu'il propose ${ }^{12}$ »); il retrouve à Syracuse (non sans être, comme par hasard, escorté par tous les "Grands " de la cité) le tombeau d'Archimède grâce à la mémoire exacte qu'il a des vers de l'épitaphe du grand homme que ses propres concitoyens ont oublié ${ }^{13}$; il raffermit, après l'acquittement de Clodius (61 av. J.-C.), le courage de ses concitoyens en n'utilisant, dans une périphrase savoureuse de fausse modestie, pas moins d'une dizaine de termes différents pour parler de lui à la fois à la troisième et à la première personne : Idem ego ille (non enim mibi uideor insolenter gloriari cum de me apud te loquor, in ea praesertim epistula quam nolo aliis legi) idem, inquam, ego recreaui afflictos animos bonorum... : "Ce fut moi encore (je ne crois pas me vanter de manière immodeste lorsque je te parle de moi, surtout dans une lettre que j'interdis de lire à d'autres), ce fut moi, dis-je, qui ai raffermi le courage des gens de bien ${ }^{14} \ldots$... On ne diminue en rien la figure du grand homme en l'humanisant et, surtout, on enrichit sa personnalité (on n'évacue pas au profit d'une unité artificielle, devenue mythique, l'éclatement d'une réalité historique et psychique) en même temps que l'analyse se focalise davantage sur la mise en scène littéraire, ses procédés et ses objectifs. L'approche des textes latins n'est en rien différente ce qui se passe dans la classe de français, mais les outils et les méthodes sont bel et bien communs.

\section{Comédie, satire, épigramme : même question}

Les $I O$ centrent l'approche des textes qui appartiennent à cette catégorie sur la question du rire, ce qui est légitime. Mais parmi les auteurs cités, après les attendus Martial et Juvénal, on trouve Pétrone et Apulée, des auteurs de romans. On ne peut certes douter que Pétrone soit comique. Mais son comique naît précisément de la transgression des règles, ce qui pose nécessairement la question de la pertinence du classement générique retenu. En effet, à Rome, le roman n'est pas constitué en genre (on ne trouve nulle part la moindre définition codifiée, seul Macrobe - mais c'est à la fin du IVe siècle - s'y est essayé de manière adventice), mais il est le " genre de tous les genres ", une satura, c'est-à-dire une " macédoine ", en même temps qu'une "satire " des temps néroniens. L'approche doit être, du point de vue des genres, transversale et se demander ce qu'est le Satiricon, à savoir " une épopée dégradée 15 ", l'aventure d'anti-héros, sans but ni idéal, et surtout sans dieux. La parodie de l'épopée est une question centrale de l'interprétation du Satiricon, et problématique. La critique ne parvient ainsi pas à s'entendre sur la signification des 295 vers composés par Eumolpe (chapitres 118-124) sur le thème de la guerre civile, inspirés par

Les secrets de la correspondance de Cicéron, Paris, 1947.

14 Epist. ad Att. 1, 16, 8.

15 Je renvoie aux travaux de G. LuKACS, La Théorie du roman (1920), Paris, 1971. 
Lucain (dont Pétrone écrirait un pastiche : c'est la thèse la plus ancienne, soutenue dès 1842 par J. G. Mössler) ou, sinon modèles, du moins inspirateurs de Lucain (théorie de P. Grimal, fondée notamment sur des arguments chronologiques) 16 .

Le théâtre latin (Plaute et Térence sont rangés par les $I O$ dans ce chapitre) n'est pas non plus un genre pur. En interrogeant, en 2002, les candidats à l'oral de l'agrégation interne sur l'Andrienne de Térence, j'ai été surpris par l'approche systématisante des explications entendues. Soit, aux dires de certains candidats, tout était comique dans la pièce. C'est naturellement faux, on rencontre dans la pièce des passages pathétiques et dont la tonalité est originale (mort de Mysis, sœur de l'Andrienne), mais certainement ni vaudevillesque, comme on l'a prétendu à tort, ni proche de la comédie de Boulevard. Soit, aux dires d'autres, la comédie virait à la tragédie, ce qui est un autre contresens. Ces exemples montrent la difficulté qu'il y a à cerner la tonalité du théâtre de Térence et à le ranger unilatéralement dans un seul genre : n'a-t-il pas inspiré aussi bien Molière (le Phormion est imité dans les Fourberies de Scapin) que Diderot (qui a utilisé l'Hécyre), été ainsi aux origines de la farce comme du drame bourgeois ? César ne le jugeait qu’à moitié comique (il évoquait sa semi-uis comica). Peut-être gagnerait-on alors à privilégier une approche historico-littéraire et à centrer la réflexion autour d'une interrogation telle que : "Place et représentation de l'esclave à Rome ", ce qui rend sans doute les choses plus complexes mais aurait l'avantage d'éviter le manichéisme traditionnel d'une approche trop scolaire montrant en Rome une civilisation cruelle et esclavagiste. Aussi bien Trimalcion que Dave (Andrienne) ont une place dans cette société qui évolue. L'esclave de Térence, nourri d'aristotélisme ${ }^{17}$, philosophe sur les relations maitre/esclave ${ }^{18}$; le parvenu de Pétrone cherche à se faire une place dans la société impériale. La prise en compte de l'évolution historique des temps (la fin des guerres puniques et l'influence intellectuelle du cercle des Scipions dans un cas, l'évolution du régime impérial et des cadres sociaux sous Néron dans l'autre) ne peut être disjoint de l'approche générique : la rigidité de cette dernière doit être tempérée par l'analyse contextuelle.

\section{Histoire et épopée : un vrai choix transgénérique ?}

Cette fois, le titre de la rubrique des $I O$ fait un choix explicitement transgénérique. On semble ici encourager les comparaisons entre le traitement d'un même épisode (mythologique ou historique) dans deux ouvres qui appartiennent à deux genres différents. J'ai eu le plaisir de retrouver dans ce passage des $I O$ la suggestion d'explorer la piste que j'avais suivie dans un article publié en $1996{ }^{19}$ et qui comparait le traitement de l'épisode du passage du Rubicon chez Lucain et

16 Cf. P. GRIMAL, "Le Bellum ciuile de Pétrone dans ses rapports avec la Pharsale", Rome, La littérature et l'histoire, Paris, 1986, p. 135-143.

17 Cf. P. Grimal, "Térence et Aristote. À propos de l'Héautontimorouménos", Rome, La littérature et l'histoire, Paris, 1986, p. 747-760.

18 On relira le dialogue entre l'affranchi Sosie et son ancien maître Simon aux vers 34-45 de l'Andrienne; Sosie, absent, si l'on en croit Donat, des pièces de Ménandre dont s'inspire Térence, offre nécessairement par sa présence incongrue une clef de la pièce.

19 "Le Rubicon illustré dans l' Histoire Romaine de Mansel", dans Les manuels scolaires de langues anciennes, Annales Littéraires de l'Université de Besançon, éd. St. RaTTi, Besançon, 1997, p. 125-145. 
chez Suétone. J'avais, à l'époque, tenté de montrer comment une enluminure de l'Histoire romaine de Mansel (XVe s., inédit), publiée dans un manuel de latin pour une classe de 4 e, montrant César traversant le Rubicon, avait été influencée à la fois par des notations empruntées à Suétone et, indépendamment, à Lucain.

Pour affiner l'interprétation de l'image, il faut recourir aux textes, bien sûr à Suétone et à Lucain - César ne dit mot du Rubicon. Voici le texte de Suétone :

"Comme il (César) hésitait, il reçut un signe d'en haut. Un homme d'une taille et d'une beauté extraordinaires apparut soudain, assis tout près de là et jouant du chalumeau (...). Il prit une trompette à l'un des soldats, s'élança vers la rivière et, sonnant la marche avec une puissance formidable, passa sur l'autre rive. Alors César dit : "Allons où nous appellent les signes des dieux et l'injustice de nos ennemis. Le sort en est jeté" (Iacta alea est) ${ }^{20}$."

Notre image doit donc beaucoup à Suétone et dans la miniature, comme dans les Douze Césars, l'apparition encourage César et le détermine à franchir le fleuve.

Les choses sont un peu différentes chez Lucain. Voici le texte de la Pharsale:

"Quand on vint sur les bords étroits du Rubicon, le chef crut voir le fantôme gigantesque de la Patrie en émoi ; brillant dans l'obscurité de la nuit, le regard affligé, elle avait répandu autour de son front couronné de tours ses cheveux blancs épars dont elle arrachait les mèches ; elle se dressait les bras nus et disait ces mots entrecoupés de sanglots : "Où allez-vous encore (Quo tenditis ultra), où portez-vous mes enseignes, soldats ? Si vous marchez avec le droit pour vous, en citoyens, c'est jusque-là que vous pouvez venir" 21 ."

Autrement dit, chez Lucain, l'apparition cherche à retenir César, à lui rappeler l'illégalité de sa décision. La Patrie de Lucain résiste à l'envahisseur césarien. Il y a antinomie totale avec Suétone. De quel côté est le personnage de l'illustration ? Plus vraisemblablement du côté de Suétone, donc de César.

Mais Lucain n'est pas étranger à l'élaboration de l'image : il l'a même très certainement influencée, comme le prouve le cheval dans la rivière. Lucain est le seul à présenter le Rubicon comme un fleuve redoutable dont les eaux étaient précisément gonflées au moment de la traversée de César (tumidum per amnem ${ }^{22}$ ). C'est un exploit de César qui permet finalement le passage à gué alors que le Rubicon est gros de la fonte des neiges : " D'abord le coursier au sabot sonore forme barrière contre le courant transversal et reçoit l'impulsion des ondes ; puis le reste de la foule, par un gué accessible, rompt sans peine les eaux du fleuve déjà brisé ${ }^{23}$. " Lucain a visiblement inspiré l'artiste : le courant s'accélère ; les eaux du fleuve sont constituées de lignes parfaitement dessinées et qui s'écartent de part et d'autre du cheval qui fait barrage alors que la troupe suit.

L'image emprunte donc l'une des caractéristiques du genre littéraire auquel appartient la Pharsale, je veux dire le grossissement épique. On sait que Lucain, qui écrit une épopée historique, dont les événements, connus de tous les lecteurs contemporains, dataient à peine d'une petite centaine d'années, se heurtait, dans son entreprise d'écrire une véritable épopée, à deux difficultés majeures : ses personnages ne sont pas légendaires et les dieux n’interviennent pas dans

Vie de César 32.

Pharsale I, 185-192.

Pharsale I, 204. 
la Pharsale, au contraire de ce qui se passe dans l'Énéide. Pour pallier le second obstacle, Lucain a recours au merveilleux, qui se substitue au divin, et pour contourner le premier, il grossit délibérément le moindre événement pour le hausser à la hauteur de l'épopée. Comme l'écrivait Désiré Nisard, "Lucain fit les batailles plus meurtrières, les soldats plus féroces, les pertes d'hommes plus grandes ; il convertit les ruisseaux de sang en rivières, les escarmouches en combats, les collines en montagnes 24 ». Notre image a donc une dimension épique et il convient de la faire lire comme telle à des élèves.

Mais un regard plus aigu sur les textes s'impose. La différence de tonalité par rapport à Suétone est patente. Chez ce dernier, César accomplit un acte bien peu glorieux : il est accompagné d'une troupe maigrichonne (modico comitatu ${ }^{25}$ ), se déplace sur une charrette attelée de mules volées à une boulangerie voisine (mulis e proximo pistrino ad uehiculum iunctis ${ }^{26}$ ), il perd son guide, de nuit, et s'égare (diu errabundus ${ }^{27}$ ), pour finir par franchir la rivière sur un petit pont (ponticulum 28). Chez Lucain, le fleuve en crue est gros de la fonte des neiges, ce qui, comme le notent les éditions savantes, est bien surprenant à cette époque de l'année ( 25 novembre ou 17 décembre 50 du calendrier julien). Et puis se pose la question du héros. La crue n'est ni chez Suétone, ni chez les grecs (Plutarque, Appien, Dion Cassius). Lucain pourrait bien l'avoir inventée pour une autre raison encore : les éléments furieux dramatisent certes l'épisode mais suggèrent aussi l'hostilité de la Nature à l'entreprise illégale du conquérant. César n'est pas le héros de Lucain, pas plus d'ailleurs que son rival Pompée qui a au moins le tort, dans cette histoire, d'être le perdant. Le vrai héros de La Guerre Civile, je veux dire le poème de Lucain, c'est Caton d'Utique.

On le voit donc, l'historiographie (en l'occurrence le genre biographique) et l'épopée, dans le cas du Rubicon, n'ont pas les mêmes exigences - même si un illustrateur inconnu a su en faire une synthèse. Suétone reprend à la suite du conquérant des arguments qui le justifient, tandis que Lucain avait besoin d'un héros à la hauteur du genre quil avait choisi d'illustrer. Beaucoup d'autres exemples pourraient être apportés de la perméabilité entre les genres évoqués. Tite-Live et Ammien Marcellin fourniraient de nombreux arguments à l'appui de la formule de Quintilien qui affirme que l'historia est proxima poetis : grossissements épiques divers qui font, chez le premier, penser que le siège de Véies est une autre guerre de Troie et, chez le second, que l'empereur Julien est un nouvel Achille. L'Énéide a par moment une tonalité tragique, qui affleure dans le récit de la mort de Laocoon au chant 2 (célèbre passage qui, du point de vue de la narratologie, est aussi un bel exemple de description en action : la frontière récit/discours/description n'est jamais étanche). Historiographie et tragédie composent encore parfois des tableaux mixtes du point de vue générique : il en est ainsi du récit de la mort de Galba par Tacite ${ }^{29}$ qui cherche à susciter chez le lecteur terreur (devant le comportement barbare de Romains égarés) et pitié

24 Études de mours et de critique sur les poètes latins de la décadence, cité dans Lucain, Silius Italicus, Claudien, éd. D. NISARD, "Collection des Auteurs Latins ", Paris, 1871, p. 11. Vie de César 31, 2.

Ibid.

Ibid.

Vie de César 31, 3.

Histoires I, 40-41. 
(devant l'affaiblissement pathétique de l'institution impériale) : ces concepts aristotéliciens sont alors plus féconds que la notion de genre.

La notion de genre littéraire appliquée à la littérature latine est pédagogiquement opérationnelle à condition de se munir de garde-fous et d'avoir à l'esprit les réserves que j'ai formulées : les emprunts, les contaminations, les transgressions, les parodies caractérisent souvent mieux telle page que l'inscription dans le genre proclamé par les titres ou les préfaces ${ }^{30}$.

\section{Conclusion méthodologique}

La démarche originale des $I O$, depuis leur profonde réforme en 1995, consiste à faire de l'élève un lecteur actif et à placer les textes (authentiques et parfois traduits) au point de départ de toute démarche. Il s'agit là d'une véritable révolution copernicienne consistant à placer l'exercice de traduction non plus à l'horizon ultime (et d'ailleurs jamais atteint) de la classe de latin, mais en son point de départ. Mieux : la traduction permet la lecture (au sens de l'analyse littéraire) des textes alors que dans l'ordre ancien des priorités une traduction, souvent laborieuse, ne permettait pas la compréhension des œuvres parce qu'elle demeurait parcellaire et myope. Une pareille démarche inductive peut être privilégiée aussi dans l'approche des genres et de leur définition. On peut ainsi partir des premiers vers de l'Énéide pour faire recenser par les élèves les caractéristiques de l'épopée :

Arma uirumque cano, Troiae qui primus ab oris
Italiam fato profugus Lauiniaque uenit
Litora, multum ille et terris iactatus et alto
Vi superum, saeuae memorem Iunonis ob iram,
Multa quoque et bello passus, dum conderet urbem
Inferretque deos Latio, genus unde Latinum
Albanique patres atque altae moenia Romae
31.

"Je chante les combats et ce héros qui, le premier, des rivages de Troie, s'en vint, banni du sort, en Italie, aux côtes de Lavinium : longtemps il fut le jouet, et sur terre et sur mer, de la puissance des dieux supérieurs, qu'excitaient le ressentiment et le courroux de la cruelle Junon ; longtemps aussi il eut à souffrir les maux de la guerre, avant de fonder une ville et de transporter ses dieux dans le Latium : de là sont sortis la race Latine, les pères Albains et les remparts de la superbe Rome ${ }^{32}$."

Quelles sont ces caractéristiques?

- l'épopée célèbre les vertus guerrières d'un héros ("l'épopée est le vrai de la guerre ", Alain) : arma / uirum / bello passus;

30 On se reportera aux trois premiers chapitres - qui font un état de la question - de l'ouvrage de G. GenetTe, Palimpsestes, Paris, 1982, p. 7-19.

32 Traduction M. RaT, Paris, 1965. 
- l'épopée fait la part belle aux interventions divines : fato / Vi superum / Iunonis;

- l'épopée illustre un dessein collectif et national : dum conderet urbem.

Les éléments ainsi recueillis pourront servir à vérifier ailleurs si telle page de Lucain répond aux mêmes exigences et, si ce n'est pas le cas, pour quelle raison. Un travail de cette nature mérite le nom de lecture (méthodique) et ne passe pas forcément par l'exercice de version si l'on a pris soin de fournir une traduction et d'éclairer un minimum le texte original. Le principe méthodologique est bien connu : les questions que pose un professeur (et un bon sujet de Baccalauréat) doivent guider l'interprétation. Les bonnes questions mettent en évidence les caractéristiques formelles du genre illustré. Par exemple, la lecture de telle page de Columelle ${ }^{33}$, qui décrit avec précision le plan type d'une villa romaine, mettra en évidence l'abondance des subjonctifs d'ordre, des futurs et des adjectifs verbaux qui ont un sens d'obligation. On fera ainsi découvrir aux élèves que le traité de Columelle s'inscrit dans le genre didactique du traité d'agronomie dont on dégagera les objectifs pratiques mais aussi idéologiques (développement de l'agriculture italienne afin d'affranchir Rome de la dépendance vis-à-vis des provinces importatrices de blé). Ou encore, on attirera l'attention sur l'abondance des subjonctifs dans l'élégie $1 \mathrm{du}$ livre $1 \mathrm{de}$ Tibulle en expliquant comment le genre de l'élégie relève d'une construction subjective : elle construit un monde de l'imaginaire, une espèce d'Arcadie mythique qui n'a rien à voir avec le monde de la guerre et de la politique augustéenne. L'analyse des modes et du lexique permettra de dégager la marginalité des élégiaques, ces contestataires des valeurs civiques traditionnelles de gloire, de richesse et d'activité au service de la Cité, et de définir leurs antonymes latins (infamia, paupertas, inertia). Voilà comment la forme permet de dégager des significations et la grammaire du sens; voilà la direction résolument moderne qu'une réflexion sur les genres, partie des canons, permet de suivre.

Mais les $I O$ contiennent d'autres pistes encore plus stimulantes.

\section{L'héritage}

Il est en effet écrit dans les $I O$ de la classe de Seconde : "Les langues anciennes permettent de comprendre l'importance du monde gréco-romain dans notre culture politique, historique, morale, littéraire et artistique " (Préambule). Je voudrais à présent proposer quelques réflexions sur les formes que prend cet héritage et qui ne sont pas précisées par les $I O$. Je privilégierai trois directions :

\section{La survie des cadres littéraires antiques dans la littérature française}

Je trouve tout d'abord fort saine et absolument fondamentale l'affirmation réitérée par les $I O$ que les méthodes d'approche des textes sont identiques en langues anciennes et en littérature française. Il y a longtemps que l'Université a intégré dans sa pratique pédagogique les méthodes d'approche modernes des textes (outils linguistiques, formes de critique héritées du structuralisme ou des recherches des dernières décennies). La notion d'intertextualité est largement répandue et 
utilisée dans les cours de nos Facultés, même si parfois elle prend les dehors plus austères de la Quellenforschung. La comparaison de textes d'époque et de genre différents sur un épisode donné figure parmi les exercices récurrents de l'enseignement supérieur et il le fait ès qualités puisqu'il s'agit pour lui d'investir dans le domaine pédagogique les résultats de recherche qui s'appuient exactement sur les mêmes méthodes.

Les $I O$ avaient mis au programme des classes de Terminale, en 2002-2003, l'histoire de Sophonisbe ${ }^{34}$. Massinissa, le vainqueur de Syphax, rencontre Sophonisbe, son épouse, la demande en mariage et lui fait porter, le même jour, du poison sur l'ordre de Scipion. Ce détail n'avait pas échappé au poète Jean Mairet, auteur en 1634 d'une Sophonisbe qui passe pour la première pièce à avoir respecté la règle des trois unités (Le Cid est de 1636) : «Massinissa, en un jour, voit, aime et se marie. »Quel meilleur exemple peut-on fournir de la permanence et de la transformation des cadres formels?

Pour revenir à Cicéron et à la rhétorique, mais sous un autre angle, il faut se rappeler que la définition des différentes parties du discours par l'auteur de l'Orator, du Brutus et du De oratore irrigue encore aujourd'hui les manuels et les pratiques d'écriture. Les traités cicéroniens de rhétorique définissent les tâches de l'orateur pour assurer sa formation, sa doctrina. Ces traités manifestent une obsession du classement en partie empruntée à Aristote ${ }^{35}$. Le discours est le produit d'une succession d'opérations dont chacune fait appel à une technique propre. Un discours est comme une dissertation (même si, historiquement, c'est l'inverse qui s'est passé) : commencer, argumenter, finir. La " mystique du plan » (G. Genette) en dissertation est un héritage cicéronien, de même que les lois du genre épidictique. Les cinq actes que doit remplir l'orateur correspondent à un bon cours de méthodologie de l'expression écrite : l'inuentio (inuenire quid dicas) n'est autre chose que la recherche des idées ; la dispositio (inuenta disponere) est l'équivalent du plan; l'elocutio (ornare uerbis) celui du style. Les deux dernières opérations ne sont pas tout à fait sur le même plan, mais correspondent exactement aux recettes enseignées pour réussir un bon exposé oral : l'actio (agere et pronuntiare), c'est jouer physiquement son texte comme un bon acteur, avec le geste, la diction et la présence physique adaptée à son auditoire, sa classe ou son jury ; l'exercice de la mémoire, memoria (memoriae mandare), permet de s'arracher à ses notes. Qui ne reconnaît dans ces prescriptions anciennes les conseils donnés à tout élève qui prépare une soutenance ou un oral ?

\section{L’appropriation de l'héritage païen par le christianisme}

La question de l'enseignement du fait religieux à l'École a beau être à la mode, comme en attestent un colloque récent tenu en Sorbonne et un rapport demandé par le Ministère de l'Éducation nationale 36 , je n'ai presque rien trouvé dans les $I O$ en relation avec la littérature latine chrétienne ou l'histoire du christianisme (en dehors de l'encouragement à lire la Bible dès le collège). Il est vrai que les règnes de Trajan et d'Antonin le Pieux constituent le butoir au-delà duquel il ne paraît pas convenable d'égarer les élèves de collège ou de lycée. La faute en incombe

Tite-Live XXX, 12-17. 
certainement à une attitude aujourd'hui dépassée qui croyait à une décadence des lettres après Tacite et Pline le Jeune. Mais on trouve encore dans les classes ce manuel ${ }^{37}$ qui, structuré de manière chronologique, arrête brutalement son cours à la mort de Marc Aurèle et passe à la trappe le dernier des Antonins, Commode, mort en 192. Si ses auteurs avaient de bonnes raisons de censurer le règne du mauvais fils que fut Commode, il faut bien admettre que, pour nombre de manuels, Constantin n'existe pas et que la question de la christianisation de l'Empire (la date de 312 est-elle pertinente ? quid de la résistance païenne dans l'historiographie latine prosénatoriale de la fin du IVe siècle ?) n'est que très rarement posée. Relevons encore que saint Jérôme n'est pas mentionné par les textes officiels et que si saint Augustin l'est, c'est en passant, par les $I O$ de Première, dans le chapitre "Expression de soi et choix de vie ", et par les $I O$ de Terminale, cette fois dans le chapitre sur la dégradation des spectacles du cirque : on n'évoque donc jamais le théologien, mais plutôt l'inventeur de l'autobiographie.

Il est bien entendu que l'approche des textes sacrés ou des Pères de l'Église ne peut se faire dans les classes que sous l'angle de la littérature et non en rapport avec une quelconque vérité de l'ordre de la foi. Cette précaution prise, il me semble que plusieurs directions de lecture peuvent être suivies afin de mettre en évidence la réappropriation chrétienne de l'héritage païen. J'en indique quelques-unes.

\section{a. Les cadres et les formes du discours}

Transmis par l'institution scolaire, ils perdurent à l'époque du christianisme triomphant. On doit se rappeler que Jérôme 38 voyait, dans un cauchemar, Dieu lui reprocher d'être plus cicéronien que chrétien. Sa Chronique est un parfait exemple de la manière de procéder des chrétiens. Publié en 381 ou 382, ce résumé de l'Histoire universelle (d'Abraham à la bataille d'Andrinople en 378), qui est pour une grande part, mais non exclusivement, la traduction d'une œuvre grecque d'Eusèbe, est un texte d'essence polémique qui fait appel aux armes mêmes des païens (Tite-Live ou les sources païennes post-liviennes) qu'il retourne contre eux. Jérôme, en s'appuyant sur les autorités du camp adverse, démontre l'antériorité des figures bibliques sur les héros de la mythologie, par exemple celle de Moïse qu'il place dans ses tableaux chronologiques en 1592 avant J.-C., quelques années avant Héraclès, placé en 1574. Les ajouts de Jérôme au texte grec d'Eusèbe sont en outre des emprunts à divers auteurs païens, dont Suétone. C'est l'insertion savante de ces ajouts dans la trame chronologique brodée par Jérôme qui les oriente dans un sens chrétien et façonne la dimension apologétique de l'œuvre. Le procédé ressemble à celui qu'Orose, au début du ve siècle, mettra en ouvre, à la demande d'Augustin, dans les sept livres de son Histoire contre les païens. La lecture de la littérature chrétienne peut et doit être faite dans la perspective du remploi polémique et de l'adaptation orientée dans un sens apologétique.

\section{b. La polémique entre chrétiens et païens}

Dans le premier livre de l'Ab Vrbe condita (I, 8), Tite-Live défendait une vision de Rome comme un lieu qui, à l'origine, avait accueilli avec générosité les étrangers et les exilés, qu'ils soient de

37 Invitation au latin 3e, Magnard, sans date de copyright. L'exemplaire consulté signale un dépôt légal en 1989.

Epist. 22, 30. 
statuts libre ou servile, en leur servant d'asylum ${ }^{39}$. Plus tard, l'historiographie chrétienne, à partir de saint Jérôme, saura se souvenir de ce motif, mais il lui servira à dénoncer le cynisme des païens, prêts à recourir aux alliances les moins recommandables : ob asyli inpunitatem magna Romulo multitudo coniungitur (" en raison de l'impunité accordée au titre de l'asile, une grande foule se trouve rassemblée par Romulus ") affirme le premier Jérôme ${ }^{40}$, que reprend Orose ${ }^{41}$, beaucoup plus critique encore : sceleratorum manum promissa inpunitate collegit (" il rassemble une troupe de bandits après les avoir assurés de l'impunité »). On peut poursuivre la lecture de Tite-Live en montrant ce que les chrétiens en ont fait : les premiers habitants de la grande Rome n'étaient que des gens de sac et de corde. L'on déforme ou l'on se réapproprie. Ce dernier cas est illustré par l'Énéide de Virgile dans laquelle les chrétiens de la fin du IVe siècle puisent au hasard des vers qu'ils interprètent dans un sens conforme à leur foi. Ces centons virgiliens sont dénoncés avec virulence par Jérôme, mais la pratique paraît avoir connu un certain succès.

Je ne résiste enfin pas au plaisir de donner un dernier exemple emprunté à une œuvre rédigée au tournant des IVe et Ve siècles, la Psychomachie du poète chrétien Prudence. Cette épopée allégorique et édifiante débute par le vers que voici :

Christe, graues hominum semper miserate labores...

"Ô Christ, toi qui as toujours eu pitié des lourdes peines des hommes... »

Or ce vers ne se comprend et ne se peut apprécier pleinement que si l'on sait qu'il est en réalité une réécriture d'un vers de l'Énéide 42 dans lequel Énée adresse une prière à Apollon :

Phoebe, graues Troiae semper miserate labores...

"Ô Phébus, toi qui as toujours eu pitié des lourdes peines de Troie..."

Comme on s'en est aperçu, les modifications opérées par Prudence, dans le respect de la métrique, sont nulles du point de vue de la syntaxe et modestes du point de vue lexical : Christe supplante Phoebe et hominum, Troiae. Outre sa parfaite connaissance de Virgile (qui était partagée par ses lecteurs) et sa virtuosité, Prudence affiche sa volonté d'effacer le paganisme (Phoebe) par le christianisme et met en application son projet qui consiste à créer, en lieu et place de l'épopée classique, une épopée militante d'un genre nouveau. Mais Prudence n’a pas eu besoin de dénaturer profondément les intentions de Virgile, car ce qu'on peut appeler une philosophie de la compassion s'illustre de manière identique chez les deux poètes. Simplement, au particularisme géopolitique qui marque l'épopée virgilienne (Troie) succède l'universalisme de la miséricorde du Christ (hominum).

39 Cf. mon étude " $A d$ coeptam magnitudinem : quelques remarques sur Tite-Live 1, 8", L'Information littéraire 5, novembre-décembre 1997, p. 3-8.

41 II, 4, 3.

42 VI, 56. 


\section{L'héritage politique : les questions de citoyenneté}

\section{a. La civilité}

La question de la Citoyenneté fait l'objet, aujourd'hui, en France, d'un regain d'intérêt. Comme souvent en pareil cas, le concept finit par perdre de sa netteté et sa spécificité par se dissoudre. Nous en sommes pour ainsi dire au point que tout à présent prend une coloration citoyenne. $\mathrm{Ne}$ parle-t-on pas de "l'entreprise citoyenne ", de l'école " citoyenne " ou mieux encore de l' " incivilité » de ses élèves ou de l'" incivilité » des banlieues ? Or, si la cité est une forme d'organisation politique des sociétés humaines qui est apparue très tôt, dans une aire culturelle et linguistique qui ne se limite pas au monde grec ni même au monde indo-européen, il demeure qu'Athènes et Rome passent pour des modèles de polis et de ciuitas et que leur influence sur les systèmes modernes est indéniable. Le terme de " civilité » connait aujourd'hui un regain de popularité sans que l'on se doute toujours que la notion latine de ciuilitas n'a cessé, pendant six siècles, d'être un terme clef de la propagande impériale romaine, du fondateur du Principat, Auguste, au Roi des Goths, Théodoric (tel que Cassiodore en dessine la figure dans les Variae ${ }^{43}$ ). Dans l'historiographie latine, qualifier de ciuilis un Prince, c'est lui reconnaitre l'une des qualités essentielles du bon gouvernant aux yeux de l'aristocratie sénatoriale traditionnelle : celle de ne pas chercher à s'élever au-dessus des autres magistrats et, par conséquent, de demeurer un citoyen ordinaire. Ce n'est pas pour d'autres raisons qu'Auguste a toujours refusé tout autre titre que celui de princeps, de " premier " sénateur, et c'est sur ces bases que s'est échafaudée la fiction du principat à la tête duquel ne règne ni un roi ni un despote, mais un magistrat parmi d'autres, distingué simplement au titre du primus inter pares.

On doit donc reconnaître que les interrogations modernes sur la citoyenneté ne peuvent se dispenser du détour par les sources antiques, ce qui prouve le caractère obligé de l'histoire et des langues anciennes ${ }^{44}$. Comment pourrait-on se passer de ces disciplines quand le ministère de l'Éducation nationale écrit que tout élève de lycée, depuis la classe de Seconde, bénéficie désormais, par an, de huit séquences de deux heures d'" éducation civique, juridique et sociale " (ECJS) 45 ? Le programme de cette discipline est édifiant : " De la vie en société à la citoyenneté »; "Institutions et pratiques de la citoyenneté "; " La citoyenneté à l'épreuve des transformations du monde contemporain 46 ». En Seconde, l'étude portera sur les notions suivantes : "Citoyenneté et civilité » (encore la ciuilitas!) ; "Citoyenneté et intégration » ; "Citoyenneté et travail 47 ".

43 Cf. mon article "Évolution et signification de la ciuilitas au VI siècle, d'Ennode à Cassiodore", dans Antiquité et citoyenneté (Actes du colloque international de Besançon), éd. St. RatTi, Besançon-Paris, Presses Universitaires Franc-Comtoise, 2002, p. 163-176. Cf. les vingt-quatre études réunies dans le volume cité à la note précédente (d'Homère à Cassiodore et des tragiques grecs à Boèce). Bulletin Officiel $\mathrm{n}^{\circ} 5$ du 5 août 1999 (Programme des Lycées).

Ibid., p. 6.

Ibid., p. 7. 


\section{b. La citoyenneté}

Un débat récent en France a prétendu opposer deux conceptions de la citoyenneté que l'on a voulu faire remonter à l'Antiquité en les affublant de noms latins : le ius sanguinis et le ius soli. On a ainsi prétendu que le droit du sol était plus libéral et serait un héritage de la révolution ; le ius sanguinis serait, quant à lui, un héritage romain. Tout cela est faux. Ces termes sont, en effet, des inventions de juristes de l'Ancien Régime. Le droit du sol remonte en fait au système seigneurial et féodal (ce qui est sur la terre appartient au seigneur) et la Révolution a voulu abolir ce système. Le ius sanguinis n’a lui absolument rien de romain : l'octroi de la citoyenneté pour les Romains est un simple acte juridique et le sang ne joue dans cette affaire aucun rôle. Ce système permet de transformer en Romain un ancien esclave comme un Grec d'origine (le poète Archias) ou un Africain. C'est ce qui fait que la citoyenneté romaine est extensible à l'infini, ce qu'avait parfaitement pressenti l'empereur Claude, à l'origine de l'élargissement des prérogatives sénatoriales aux habitants des provinces de l'Empire, en commençant par ceux de la Gaule ${ }^{48}$. À ce titre, l'édit de Caracalla, en 212, n'est que l'aboutissement si naturel d'un processus entamé depuis plusieurs siècles (peutêtre même depuis l'asylum romuléen) que personne, à l'époque, parmi les historiens, n’a cru bon de s'appesantir sur ce qui n’a pas été un événement pour les contemporains.

\section{Interprétation, idéologie et lecture des textes}

\section{La lecture : essai de définition}

La grande réforme, me semble-t-il, opérée ces dernières années par les $I O$ relève d'un changement radical et salutaire de perspective : on ne cherche plus à former des élèves traducteurs. La version n'est plus l'exercice roi autour duquel tourne toute la constellation des gammes préparatoires. Lobjectif est de mettre les élèves en position de lecteurs et on place désormais au centre du système la lecture des textes authentiques, le plus tôt possible, et ce en langue originale, avec l'aide occasionnelle et intelligente des traductions (ou des comparaisons de traductions). Les nouvelles épreuves de Baccalauréat, mises en place depuis 1995, confirmées en 2004, entérinent ce changement d'objectif.

Mais qu'est ce que lire un texte antique ? J'avancerai prudemment, et dans le simple but de nourrir le débat, l'esquisse de définition suivante : il pourrait s’agir de mettre en évidence les motifs idéologiques, religieux ou politiques au service desquels l'auteur place les ressources formelles et rhétoriques quil mobilise. Si les techniques d'analyse appliquées aux textes anciens sont les mêmes qu'en français, il subsiste en revanche une énorme différence entre la littérature latine (c'est moins vrai, je crois, pour la littérature grecque) et la littérature française : la première ignore la notion d'art pour l'art et obéit toujours à des préoccupations moralisantes, voire édifiantes. Le beau n'y sourd que par surcroit, ce que Stendhal avait pressenti en affirmant, à propos des monuments de Rome, que le "beau n'était que la saillie de l'utile " (Promenades dans Rome).

48 Sa position et ses arguments nous sont connus par la Table Claudienne de Lyon et par Tacite, Annales XI, 24 qui résume, à sa manière, le discours de l'empereur tenu au Sénat en 48 . 


\section{Une histoire rhétorique}

Le plus simple est de prendre le domaine de l'historiographie. Il me paraît nécessaire de faire comprendre à des élèves à quelles règles obéit l'historiographie antique.

L'histoire à Rome n'est jamais simplement une narration. Si cette affirmation est devenue une évidence pour des modernes que la critique littéraire a convaincus que toute transposition d'une réalité dans les mots engage une interprétation, elle ne l'était pas pour les lecteurs des œuvres antiques, jusqu'à il y a peu. Trop d'historiens notamment ont pris au pied de la lettre l'affirmation de Quintilien selon laquelle " on écrit l'histoire pour raconter et non pour prouver" (historia scribitur ad narrandum, non ad probandum) ${ }^{49}$. Tout montre au contraire que l'historiographie latine - et même grecque, avec Fabius Pictor - , depuis les origines, depuis les annalistes de l'époque républicaine jusqu'aux abréviateurs contemporains d'Ammien Marcellin, en passant pas les plus grands, Tite-Live notamment, cherchent à démontrer une thèse, à savoir, la plupart du temps, à justifier le passé et à glorifier les actions héroïques du peuple romain 50 . À cet égard, la trop fameuse formule cicéronienne du De legibus n'a pas toujours été prise dans toute sa vraie dimension. Lorsque Cicéron affirme historia... opus unum hoc oratorium maxime ${ }^{51}$, il ne veut pas simplement dire que "l'histoire est une tâche qui revient à l'orateur " - sans doute songeait-il à sa propre personne - mais qu'il s'agit là de la plus noble des tâches de l'orateur et que ce dernier mettra au service de l'écriture de l'histoire tous ses talents oratoires, c'est-à-dire littéraires, car l'orateur est avant toute chose un écrivain.

Le talent littéraire est le premier dont doit faire preuve l'historien, qui est un écrivain avant tout, un historiographe. Les mots latins pour désigner ce talent sont eloquentia et ingenium. Le but avoué de Tacite est de rivaliser avec ses devanciers, ces eloquentissimi auctores dont parle l'Agricola $(10,1)$ et dont il exalte le talent individuel (celeberrimus quisque ingenio ${ }^{52}$ ). Le propre de la grande histoire, par opposition au genre moins noble de la biographie, illustrée par Suétone, est de se hisser au-dessus de la sécheresse des premiers annalistes pour faire œuvre éloquente. Pareille exigence explique une constante de l'historiographie antique que les modernes, notamment parmi les historiens, ont beaucoup de difficulté à accepter : l'historien a toute latitude pour présenter les faits à sa manière afin de mieux séduire et convaincre son lecteur. Tacite a bien récrit (sans en modifier la teneur de fonds) le discours de Claude 53 prononcé en 48, au Sénat, devant les notables gaulois venus demander une extension de leurs droits politiques, comme le prouve de manière indubitable la comparaison avec le discours authentique de Claude, conservé par la célèbre inscription des Tables de bronze de Lyon ${ }^{54}$. Cette licence était de fait reconnue aux historiens, ainsi que

49 Institution oratoire $\mathrm{X}, 1,31$.

50 Cf. F. PASChOUd, "Réflexions sur le problème de la fiction en historiographie", L'historiographie de l'Église des premiers siècles, éd. B. POUDERON et Y.-M. Duval, Paris, 2001, p. 23-35. De legibus I, 5.

Agricola I, 2.

53 Annales XI, 24.

54 Cf. J. CARCOPINO, "La table claudienne de Lyon et l'impérialisme égalitaire", Journal des Savants, 1930, p. 69-93, article repris dans Points de vue sur l'impérialisme romain, Paris, 1934, p. 159-199. 
l'affirme sans détour Cicéron, par la bouche d'Atticus : concessum est rhetoribus ementiri in historiis, ut aliquid dicere possent argutius 55 ( "Il a été autorisé aux rhéteurs d'affabuler dans les histoires afin de pouvoir argumenter de manière plus serrée »). Le verbe ementiri ne doit en aucun cas être traduit par "mentir ", terme qui implique une notion de faute morale absente du mot latin ici, mais plutôt par " affabuler ", " embellir », " améliorer " ou encore " colorer ».

Les moyens de l'historien ne ressortissent pas simplement à la mission d'information, ou à la raison, docere, mais aussi au sentiment, mouere, delectare. Ainsi s'expliquent les libertés prises par l'historien à des fins de dramatisation ou d'édification. L'un des exemples les plus flagrants a été récemment mis au jour grâce à la découverte d'une inscription. Il s'agit du sénatus-consulte de Pisone patre qui jette une lumière nouvelle sur le procès du prétendu assassin de Germanicus, connu jusque-là essentiellement grâce à Tacite ${ }^{56}$. Alors que Tacite place le procès au début de l'année 20, on sait désormais, grâce à l'inscription, qu'il eut lieu en réalité le 10 décembre 20. Pour quelle raison Tacite a-t-il sciemment modifié la chronologie ? Est-ce, comme l'avancent des travaux récents, afin de ne pas introduire de solution de continuité entre la mort de Germanicus et la date du procès et donc une inévitable chute de tension dramatique ? Le débat est ouvert, mais l'exemple doit nous rappeler que l'historien a une certaine liberté, notamment dans le choix des faits, leur agencement ou leur éclairage, et nous faire souvenir qu'en exerçant ce droit il use des prérogatives du rhéteur.

Les encouragements aux précautions méthodologiques que je viens d'exposer me paraissent d'autant plus fondées que je découvre dans le programme des auteurs latins pour le collège, p. 155, le nom de l'Histoire Auguste (écrit par erreur avec un a minuscule : Histoire Auguste, qui est une expression empruntée à la Vie de Tacite 57 , signifie histoire impériale, ou histoire des empereurs). On permettra à l'éditeur d'une partie de cette œuvre dans la collection "Budé " de dire qu'il est passé, à cette découverte, par trois sentiments : j’ai été surpris, puis flatté, enfin j’ai été inquiet et je le suis toujours. Il faut savoir, en effet, que l'Histoire Auguste est le plus grand canular littéraire de toute l'Antiquité, ce qui n'est pas toujours parfaitement assimilé par les profanes ${ }^{58}$. À preuve, l'œuvre se présente comme ayant été écrite sous les empereurs Dioclétien et Constantin, au tournant des III et IVe siècle, alors qu'elle date en réalité, comme l'ont établi les spécialistes, de l'extrême fin du IVe siècle. Elle se présente en outre comme ayant été composée par six biographes différents alors que son auteur est unique et qu'il se dissimule sous des pseudonymes dont le nouveau Gaffiot aligne toujours avec une naïveté scrupuleuse les noms sur le même plan que ceux de Tite-Live ou de Tacite. Il peut certes être passionnant de lire l'Histoire Auguste au collège, mais il est irresponsable de le faire sans aucune préparation, comme s'il s'agissait d'un témoignage historique fiable alors que la forgerie et le canular font peser sur l'essentiel de son information le soupçon fondé du mensonge 59 .

55

56

57

58

59

Brutus XI, 42.

Annales II, 69-70 et 3, 8 .

$\mathrm{X}, 3$.

Cf. les introductions aux volumes parus dans la "Collection des Universités de France " (coll. "Budé ») : I, 1 (J.-P. Callu, 1992) ; III, 1 (R. TurCan, 1993) ; IV, 2 (St. RatTI, 2000); V, 1 et V, 2 (F. PASCHOUd, 1996 et 2001).

Il s'agit là d'un sujet d'actualité puisque j'ai proposé lors du Colloque Histoire Auguste de Bamberg (mai 2005) une identification de l'auteur de la collection : cf. mon étude à paraître, à Bari, dans les Actes du colloque, éd. G. BONAMENTE et H. BrandT, "Lauteur de l'Histoire Auguste: une hypothèse nouvelle". 


\section{Conclusion}

Les $I O$ de langues anciennes sont donc résolument modernes et ambitieuses, ce dont on ne saurait trop se féliciter. Rien ne serait pire, en effet, pour l'avenir des langues anciennes, qu'une politique mensongère promettant des disciplines faciles et ludiques : ce serait les condamner à terme. En revanche, les travaux personnels encadrés (les TPE), en lycée, sont l'occasion de promouvoir de vrais travaux de recherche; les professeurs des lycées ont l'occasion d'exercer là une vraie responsabilité, comparable à l'encadrement d'une maittrise dans l'enseignement supérieur. Une autre chance pour les langues anciennes, à condition d'être imaginatif et innovant, sont les parcours de découverte en collège.

Afin d'engager les professeurs dans les voies de la lecture et de l'interprétation (soyons honnêtes : combien de professeurs proposent de vrais commentaires pour chacun des textes lus en cours ?), je me demande sil ne faudrait pas aller encore plus loin et encourager la lecture, en langue française, d'extraits d'œuvres latines d'une certaine ampleur - plutôt que des fragments dès le collège. On peut lire, en traduction, l'Andrienne ou Tite-Live en classe de Quatrième, comme on y étudie une pièce de Molière ou des nouvelles de Maupassant. Il existe un De Viris illustribus tardif mais authentique. On n’a donc pas besoin du De Viris écrit par Lhomond ni de son vrai-faux latin : ce manuel du XVIII siècle est un condensé d'exceptions grammaticales destiné à inculquer aux élèves des collèges jésuites les règles de la syntaxe latine. On ne peut, en outre, le commenter, car il est un simple digest de Tite-Live, expurgé de toute sa saveur littéraire. On assiste aujourd'hui à une situation paradoxale : on lit dix ou douze pages de Tite-Live en Terminale, assez péniblement, comme le montre le recours aux traductions juxtalinéaires, alors qu'on aurait pu les lire dès la classe de Troisième, beaucoup plus aisément si cette lecture avait été faite en traduction avec un équipement pédagogique adapté.

L'approche critique des textes et la quête du sens doivent prendre la priorité sur l'apprentissage simplifié d'une mythologie récréative ou d'un catalogue des empereurs romains proches de l'imagerie d'Épinal. Approche transversale, recoupements, comparaisons de textes, intertextualité : voilà les mots clefs d'un enseignement des langues anciennes qui privilégie de manière légitime les textes et les œuvres. Puisse-t-on considérer les données et les analyses qui précèdent comme une invitation au débat et les entendre dans une perspective comparatiste avec les approches littéraires ainsi que les systèmes éducatifs européens.

STÉPHANE RATTI

Professeur de langue et littérature latines

Université de Dijon

2 boulevard Gabriel - 21000 Dijon

stephane.ratti@u-bourgogne.fr 